\title{
Harnessing Effective Molarity to Design an Electrochemical DNA- based Platform for Clinically Relevant Antibody Detection
}

\author{
Marianna Rossetti, ${ }^{[a]}$ Simone Brannetti, ${ }^{[a]}$ Marco Mocenigo, ${ }^{[b]}$ Bruna Marini, ${ }^{[b]}$ Rudy Ippodrino, ${ }^{*[b]}$ \\ Alessandro Porchetta ${ }^{*[a]}$
}

[a] M. Rossetti, S. Brannetti, A. Porchetta

Department of Chemistry

University of Rome Tor Vergata

Via della Ricerca Scientifica, 00133 Rome, Italy

E-mail: alessandro.porchetta@uniroma2.it

[b] M. Mocenigo, R. Ippodrino, B. Marini

Ulisse BioMed S.r.l.

Area Science Park, 34149 Trieste, Italy

Supporting information for this article is given via a link at the end of the document

\begin{abstract}
Rapid and easy-to-use platforms for antibody detection are likely to improve molecular diagnostics and immunotherapy monitoring. However, current technologies require multi-step, timeconsuming procedures that limit their applicability in these fields. Inspired by Nature, which often achieves regulation of reaction rates and binding affinity through nanoscale spatial confinement of biomolecules, here we demonstrate effective molarity-driven electrochemical DNA-based detection of target antibodies. We show a highly selective, signal-on DNA-based sensor that takes advantage of antibody-binding induced increase of local concentration to detect clinically relevant antibodies in blood serum. The sensing platform is modular, rapid, and versatile and allows the multiplex detection of both IgG and IgE antibodies. We also demonstrate the possible use of this strategy for the monitoring of therapeutic monoclonal antibodies in body fluids. Our approach highlights the potential of harnessing effective molarity for the design of electrochemical sensing strategies.
\end{abstract}

\section{Introduction}

The production of low-cost, easy-to-use platforms for the monitoring of clinically relevant antibody level would dramatically impact many fields of application ranging from molecular diagnostics ${ }^{[1]}$ infectious disease control ${ }^{[2]}$ and therapeutic drug monitoring. ${ }^{[3]}$ The development of point-of-care testing method could allow more frequent diagnostic screening, especially in low in-come countries, and shortens the delay between sample acquisition, diagnosis and therapeutic decisions in clinical settings. ${ }^{[4]}$ To achieve these objectives, the quantitative detection of antibodies has to be rapid, single-step and deployed directly in complex matrices. To date, conventional serological methods (i.e. ELISA or Western blot assays) can be easily performed without complicated sample pre-treatment, yet they require multi-step, time consuming procedures and sophisticated instrumentations that limit their use in routine clinic diagnostics. ${ }^{[5]}$ Additionally, most of the available sensing methods for antibody detection generally fails when deployed directly in complex clinical samples. In blood serum/plasma, for example, nonspecific absorption onto the sensor surface can dramatically affect label-free methodologies that rely on the mass and refractive index monitoring (e.g. microcantilever, SPR). ${ }^{[6]}$ Label-free electrochemical sensors instead show poor selectivity in complex matrices. ${ }^{[7,8]}$ Similarly, field effect transistor sensors generally show a significant decrease of the sensitivity and detection limit in biological environments, due to the high ionic strength of the media, and only one example recently reported a FET system for the detection of antibodies operating under physiological conditions. ${ }^{[9]}$

Over the past decade, a number of DNA-based platforms showing single-step detection of antibodies have been reported. ${ }^{[10-19]}$ Among them, electrochemical DNA sensors (EDNA) hold great promise ${ }^{[20-23]}$ because they are multiplexable, calibration-free systems ${ }^{[24]}$ that require low-cost signal transduction equipment. Although E-DNA sensors stands out for wide developments towards point-of-care diagnostics of small molecules, ${ }^{[25-28]}$ nucleic acids $^{[29,30]}$ and proteins, ${ }^{[27,31-33]}$ the electrochemical detection of clinically-relevant antibodies is limited to few examples. ${ }^{[34]}$ In this regard, pioneering works of Plaxco and co-workers have demonstrated the possibility to detect antibodies in complex matrices using a rigid double stranded nucleic acid scaffold ${ }^{[35]}$ labelled with methylene blue redox reporter and an antibody-binding recognition element. ${ }^{[36-39]}$ One of the main limitation of this approach is represented by a significant nonspecific signal drift when employed in whole blood, a problem that restricts their transition as POC diagnostic sensors. ${ }^{[39]}$ Similarly, Vallée-Bélisle et al. employed antibodies as steric hindrance effectors that alter the binding efficiency and hybridization kinetic of the redox-active signalling strand to a DNA capture probe bound on the electrode. ${ }^{[40-42]}$ Unfortunately, each of those platforms operate in a signal-off fashion, limiting the gain of the sensor ${ }^{[43]}$ and also making the platform prone to false positive response due to the degradation of scaffold probe. ${ }^{[44]}$ Although many efforts have been devoted to improve the signal gain of E-DNA platforms, for instance by optimizing the frequency and amplitude of the square-wave potential ramp ${ }^{[45]}$ and the redox moiety employed, ${ }^{[46]}$ the rational design of mechanisms capable of bringing the redox element close to the electrode upon target binding is ideally suited to high-gain E-AB signalling. In this regard, an electrochemical switch-based DNA sensor have been engineered to detect antibodies in whole blood.[47] Such an approach, however, would not be easily generalizable because it 
requires a careful thermodynamic optimization of the switch and can strongly be affected by the size of the antigen.

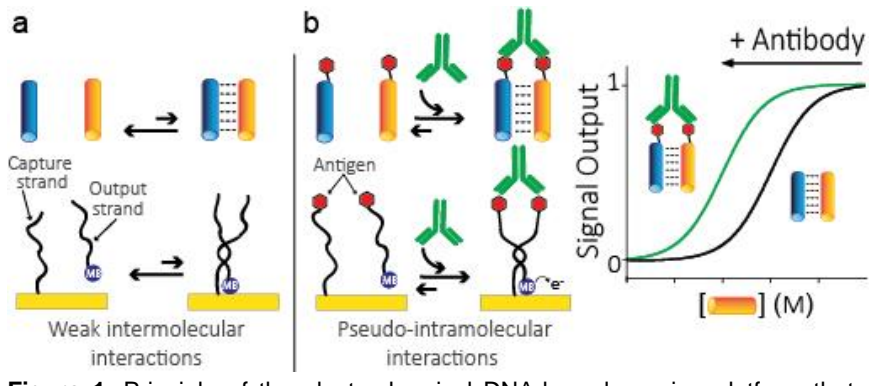

Figure 1. Principle of the electrochemical DNA-based sensing platform that employs effective molarity to detect antibodies. (a) We designed the capture and the output strand in a way that their intermolecular interactions are weak and relative binding affinity is poor. (b) The co-localization of the antigenlabelled capture and output strands on the same target antibody generates an improvement of the observed binding affinity, and brings the redox-active tag near the gold surface thus generating an increase in the current signal.

Recently, we have proposed a nucleic acid-based platform for antibody and small molecule detection that couples the advantageous features of structure-switching nucleic acid probes with those of target induced co-localization based methods. ${ }^{[48,49]}$ Although this approach allows the rapid, signal-on detection of antibodies, the fluorescence output makes the platform hardly multiplexable due the limited number of FRET pairs suitable in real matrices. Moreover, the miniaturization of the fluorescence device and its use in off-laboratory settings could prove challenging.

We propose here a signal-on, highly selective electrochemical sensor that takes advantage of effective molarity of biomolecules when confined together at the nanoscale. The concept of effective molarity is pervasive in cell biology and enzymology. Co-localization and compartmentalization of macromolecules increase effective molarity in biochemical processes such as transcription and translation, thus enhancing the speed of enzymatic reactions by several thousand-fold. ${ }^{[50,51]}$ Also protein-protein interactions and related biological functions are generally enhanced through the introduction of a physical connection (i.e. linker) between the partners, ${ }^{[52,53]}$ as an enzyme to its substrate or weakly interacting proteins to each other. ${ }^{[54]}$ This is due to the fact that the change from inter- to intramolecular interactions generally results on an increase of the encounter rates and binding affinity (Figure 1a). ${ }^{[55]}$ Inspired by this naturallyoccurring mechanism, here we employ antibodies to drive the colocalization of two antigen-labelled nucleic acid strands. Specifically, we demonstrate that the co-localization of a DNAbased capture strand anchored on the electrode with a redoxlabelled DNA strand (output strand) free in solution on the same target antibody provides a means to increase their local effective molarity and relative binding affinity (Figure $1 \mathrm{~b}$, green line). This brings the redox label (methylene blue) close to the electrode surface, producing a significant increase of the electrochemical signal upon binding of the specific target antibody.

\section{Results and Discussion}

To evaluate co-localization effect on the binding affinity between the capture and the output strand, we first studied the mechanism using a nucleic acid strand that mimics the action of the target antibody (Figure $2 \mathrm{a}$ and S1). Since the antibodymimicking strand presents two terminal portions that are complementary with both the capture and the output strands, it is able to bring them into close proximity and improve their relative binding affinity. Specifically, the Ab-mimicking strand is designed with two terminal 17-base long portions (orange portions, Figure $2 \mathrm{a}$, left) complementary with the capture and the output strand (blue portion) respectively, joined by a 14-base long poly-(T) linker in the middle (green fragment) to ensure the conformational freedom that ultimately affects the proximity effect. ${ }^{[56]}$ The capture strand is immobilized on the surface of gold screen printed electrode (SPE) via sulphur-gold chemistry, and also presents a 5 '-terminal portion complementary with the 3 '-end of the output strand (black portions). The output strand is a redox-active DNA strand labelled with a methylene blue tag at the 3 '-end. Capture and output strands are designed with complementary portions so that in the absence of the Ab-mimicking strand their binding affinity is poor $\left(\mathrm{K}_{1 / 2}=97 \pm 4 \mathrm{nM}\right)$. As expected, in the presence of Ab-mimicking strand ( $100 \mathrm{nM})$, the binding curve performed by adding increasing concentrations of the output strand showed improved relative affinity between the two strands (Figure $2 \mathrm{~b}$, red line $\mathrm{K}_{1 / 2}=18 \pm 2 \mathrm{nM}$ ).
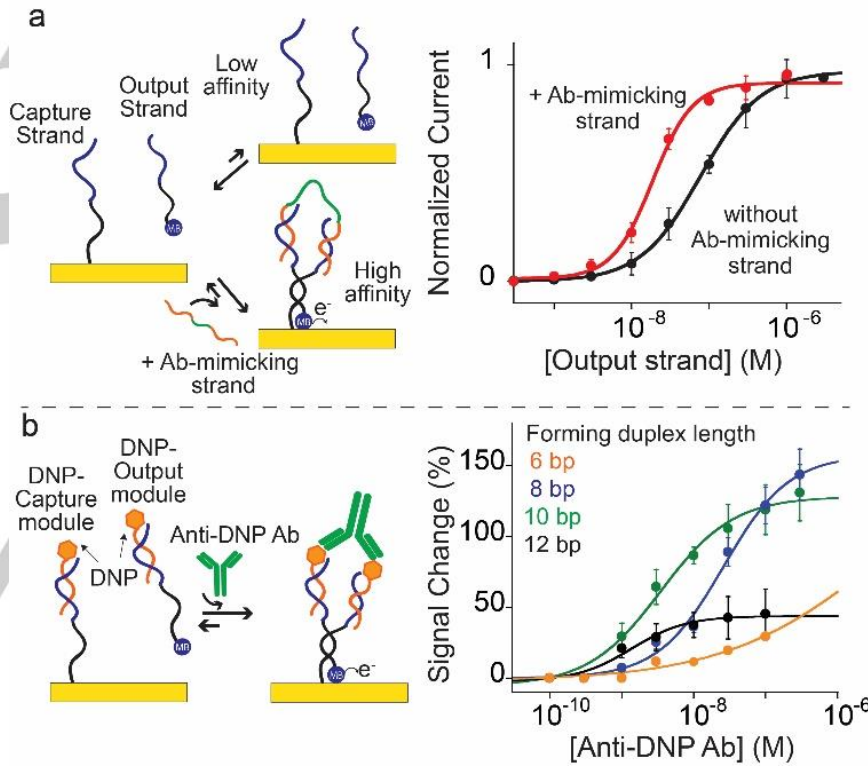

Figure 2. Design of the effective molarity-driven electrochemical DNA-based platform. (a) In the presence of an antibody-mimicking strand (100 nM) we observed an increase in the observed binding affinity (red curve) between the capture and the output strands. (b) Modular platform for anti-DNP antibody detection. We selected 2,3-dinitrophenol (DNP) as the antigen and conjugated it to a scaffold strand (orange line) fully complementary with a portion of both the capture and the output strands (blue portion). We tested DNP-output modules having different length of the complementary domain (from 6 bp to 12 $\mathrm{bp})$. The experimental values represent averages of three separate measurements and the error bars reflect the standard deviations. For further experimental detail see $\mathrm{SI}$.

As initial proof-of-principle of the method for antibody detection, we selected the small molecule 2,3-dinitrophenol (DNP) as antibody-binding recognition element (i.e. antigen) and anti-DNP $\operatorname{lgE}$ antibody as the counterpart target (Figure 2b). To make the platform more versatile, we tested a modular design that employs a third 24-base long nucleic acid strand (scaffold strand), terminally modified with the recognition element (DNP). The DNPscaffold strand (orange strand in Figure 2b) is fully complementary with both the capture and output strand (blue 
portions in Figure 2b) described above. This design allows the detection of a number of antibodies by only changing the recognition element conjugated to the scaffold strand. To achieve optimal sensitivity, we tested a set of DNP-output modules (output strand + DNP-scaffold strand, Figure $2 b$ ) with different lengths of the complementary domain (black portion, from $6 \mathrm{nt}$ to $12 \mathrm{nt}$ ). First, we performed binding curves by adding increasing concentrations of DNP-output module (output strand + DNPscaffold strand) in the presence of DNP-capture module (capture strand + DNP-scaffold strand) anchored to the gold electrode. As expected, we observed different binding affinities according with the number of complementary nucleotides (Figure S2).
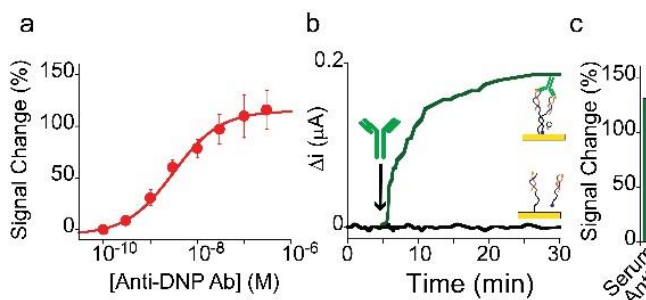

Figure 3. (a) Signal gain as function of anti-DNP antibody concentrations in serum sample. (b) Peak current of SWV measurements (each 30s) shows that anti-DNP antibody detection (100 nM) is rapid, reaching plateau of the current in less than $10 \mathrm{~min}$. (c) Specificity assays in the presence of a-specific antibodies in serum samples. Control tests performed by adding anti-DNP antibodies in the absence of the output strand (\#1), DNP-scaffold strand (\#2), and the combination of the two (\#3). All experiments were performed in $20 \%$ blood serum at $25^{\circ} \mathrm{C}$. The experimental values represent averages of three separate measurements and the error bars reflect the standard deviations.

Second, we tested the antibody sensor by adding increasing concentrations of anti-DNP antibody in $100 \mu \mathrm{L}$ solution containing a fixed amount of the DNP-output module (10 nM) (Figure 2b, right and S3). We found that the length of the duplex forming portion leading to the optimal sensitivity for antibody detection is 10 bases $\left(K_{1 / 2,10 \mathrm{bp}}=3.5 \pm 0.8 \mathrm{nM}\right)$, which has been thus selected for the successive experiments. As expected, for DNP-output modules forming shorter nucleic acid duplex ( $6 \mathrm{bp}$ and $8 \mathrm{bp}$ ), the titration midpoint was reached at higher anti-DNP antibody concentrations $\left(\mathrm{K}_{1 / 2,6 \mathrm{mer}}=\mathrm{n} . \mathrm{d}\right.$; $\mathrm{K}_{1 / 2}$, 8mer $\left.=26 \pm 6 \mathrm{nM}\right)$. On the contrary, the 12 bp-long forming duplex shows similar binding affinity $\left(\mathrm{K}_{1 / 212 \mathrm{bp}}=1.4 \pm 0.4 \mathrm{nM}\right)$ but lower signal gain. This is due to the fact that we have a significant fraction of DNP-output module already bound to the DNP-capture module in the absence of the antibody, and this generates higher current background and consequent small current changes. In our opinion, this is coherent with the fact that by increasing the length of complementary duplex forming portion we increase the amount of pre-assembled scaffold/output module complex in the absence of target antibody and this results in a signal off current output according to the collisional signalling mechanism in E-DNA platforms. ${ }^{[57]}$ To further optimize the sensing platform, we tested different concentrations of the DNP-output module (Figure S4) and selected a concentration of $10 \mathrm{nM}$ for next experiments. Then, we investigated the co-localization mechanism testing different density of DNP-capture module on gold SPE. We functionalized several electrodes with different capture module density and observed that the platform only performs properly when employing a low density (Figure S5 and S6). ${ }^{[58,59]}$

As expected, moderately packed layer of capture module ${ }^{[40]}$ (estimated average distance $d=12 \mathrm{~nm}$; see table Fig. S6) resulted in lower signal gains because the probability to have a target antibody binding to two DNP-capture modules dramatically increases. This is due to the $\lg G$ and $\lg E$ antibodies have two identical binding sites separated by approximately 10-12 nm. ${ }^{60]}$ This package density can thus lead to cooperative binding events of target antibody to two capture modules and this event does not provide any change of the output signal. Conversely, low capture module density ( $d=28 \mathrm{~nm}$; Fig. S6) results in the optimal transduction mechanism, still maintaining an adequate number of surface-bound capture module necessary to achieve low detection limits. ${ }^{[61]}$ Ultra-low density does not guarantee an adequate number of DNP-capture module and generates low current signal. Finally, we also investigated the effect of ionic strength and we found optimal analytical performance at high ionic strength (Figure S7). Under such optimal experimental conditions, we tested the sensor in complex matrices, as blood serum samples (Figure 3, S8 and S9). The anti-DNP antibody sensing platform produces a rapid increase in the signal gain $(\sim+$ $110 \%$ ) within 10 minutes in the presence of saturating concentrations of anti-DNP antibody (100 nM, Figure $3 \mathrm{~b})$ and sensitivity in the nanomolar range $\left(\mathrm{K}_{1 / 2}=3.1 \pm 0.7 \mathrm{nM}, \mathrm{LOD}=1\right.$ $\mathrm{nM}$; Figure 3a). As expected, the platform is also highly specific due to the double recognition event necessary to produce a variation of the signal output (Figure $3 \mathrm{c}$, left). Control experiments performed by adding saturating concentration of anti-DNP antibody in the absence of i) the output strand (\#1), ii) DNPscaffold strand (\#2), and iii) the combination of the two (\#3) confirm that significant electrochemical signal change is achieved only when all the three strands are present in the same solution (Figure 3c, right). About the stability of the sensor, E-DNA platforms based on thiol-gold chemistry are stable ( $>1$ month, $T$ $=4^{\circ} \mathrm{C}$ ) and suited for use in electronic sensing applications. ${ }^{[62-64]}$ In addition, we performed polyacrylamide gel electrophoresis assay on the DNA-based output modules to confirm their stability when employed in blood serum (Fig. S10).
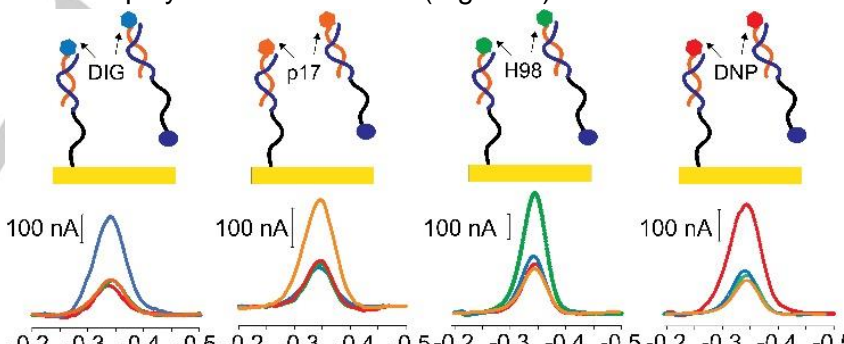

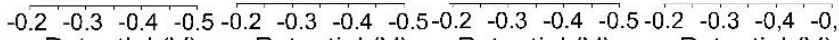

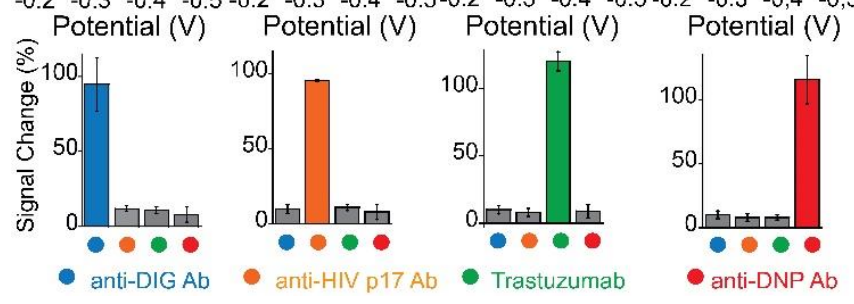

Figure 4. Orthogonal multiplex antibody detection controlled by effective molarity. Schematics of four electrodes modified with the cognate antigenlabelled scaffold strand for the simultaneous multiplex detection of anti-DIG, anti-HIV-1 p17, Trastuzumab and anti-DNP antibodies (top). Raw SWV profiles demonstrate that the current output increases only in the presence of the specific target antibody (middle). Relative signal gain obtained by adding each antibody in different combinations (bottom). All experiments were performed adding $100 \mathrm{nM}$ of target antibodies in $20 \%$ blood serum at $25^{\circ} \mathrm{C}$. The experimental values represent averages of three separate measurements and the error bars reflect the standard deviations.

To demonstrate the versatility of the strategy, we have adapted the platform for the simultaneous detection of different 
antibodies in blood serum. We have selected a set of different recognition elements ranging from small molecules (i.e., digoxigenin, DIG ${ }^{[65,66]}$ and peptides (HIV-1-p17epitope, i.e. p17;[67,68] Trastuzumab-binding mimotope peptide, i.e. H98), ${ }^{[69,70]}$ and conjugated to PNA- or DNA-based scaffold strand. Of note, hybrid DNA-PNA duplex is not sensitive to nuclease activity due to the unnatural backbone of PNA that cannot be degraded by nucleases or proteases. ${ }^{[71,72]}$ With this new set of antigen-labelled scaffold strands we were able to measure anti-DIG, anti-HIV-1 p17 and Her2-receptor targeting (Trastuzumab) antibodies respectively (Figure 4 and S11), reaching sensitivity $\left(\mathrm{K}_{1 / 2 \_ \text {Anti-DIG }}=\right.$ $3 \pm 1 \mathrm{nM}, \mathrm{LOD}=1 \mathrm{nM} ; \mathrm{K}_{1 / 2 \_ \text {Anti-HIV p17 }}=4 \pm 1 \mathrm{nM}$, LOD $=2 \mathrm{nM}$; $\mathrm{K}_{1 / 2 \text { Trastuzomab }}=9 \pm 2 \mathrm{nM} ; \mathrm{LOD}=3 \mathrm{nM}$ ) and specificity similar to those observed for the anti-DNP antibody.

Specifically, we demonstrated orthogonal multiplex detection of four different target antibodies by carrying out measurements in blood serum samples using an array of SPEs. Each gold working electrode was modified with the specific antigen-labelled scaffold strand. By adding increasing concentration of anti-Dig antibodies, only the electrochemical current of the corresponding electrode increases (+ 95\%). Similarly, testing anti-HIV-1 antibody and Trastuzumab spiked solution, the electrochemical signal increase is achieved only in the presence of the specific antibody on the corresponding electrode modified with the appropriate cognate scaffold strand. This clearly indicates that the sensing strategy is flexible and supports the electrochemical detection of all target antibodies ( $\lg G$ and $\lg E$ ) presenting antigens whose direct conjugation to a nucleic acid strand is allowed. Of note, we highlight that Trastuzumab (Herceptin $\AA$ ) is a monoclonal antibody (mAb) drug used for breast cancer treatment and for tumours overexpressing HER2/neu protein. Generally, such antibody is infused and the concentration kept in the concentration range between $35 \mu \mathrm{g} / \mathrm{mL}$ (240 $\mathrm{nM})$ and $123 \mu \mathrm{g} / \mathrm{mL}(845 \mathrm{nM})$ for an established period of time, according with the specific treatment. It has been demonstrated that Herceptin levels must be maintained above 20 $\mu \mathrm{g} / \mathrm{mL}(137 \mathrm{nM})$ in blood serum to achieve optimal therapeutic efficacy. ${ }^{[73]}$ The long half-life of antibodies in body fluids combined to the analytical features (i.e. LOD and linear range) of our platform makes our approach suitable for the selective monitoring of Trastuzumab in clinical samples of cancer-affected patients.

\section{Conclusion}

Inspired by enzyme-substrate interactions whose reaction rate and affinity can be governed by effective molarity, we have designed a highly versatile, electrochemical sensor that employs effective molarity to achieve rapid, signal-on detection of clinically relevant antibodies in blood serum. We designed antigen-labelled DNA modules in a way that the bivalent binding to the specific target antibody induces the confinement of the output module free in solution close to the scaffold module anchored on the electrode surface. The antibody binding event thus increases the effective molarity which ultimately triggers nucleic acid hybridization at the interface. This mechanism results on an increase of the electrochemical current, as function of the antibody binding, which allows its detection. We also demonstrated the possibility to monitor levels of Trastuzumab in blood serum within its therapeutic window. Since rapid monitoring and consequent personalized dosage of Trastuzumab would potentially increase immunotherapy effectiveness, we consider our platform promising for therapeutic drug monitoring in routine clinical practise.

In conclusion, it is noteworthy that our single-step direct sensing methods do not rely on any amplification step and so it cannot reach the sensitivity of standard serological clinical methods (e.g. ELISA, Western Blot methods). However, our platform appears promising for clinical and therapeutic applications because produces a rapid response (10 minutes), without multistep, wash- and reagent-intensive processes. Moreover, it is highly versatile and can be easily adapted to different antibodies by simply changing the PNA-peptide chimera probe. We also demonstrated the possibility of multiplexing using a simple array of screen printed electrodes. Standard ELISA technology instead is more complex and multiplex detection is not allowed (or at least would require a very complex optimization of the system). In addition, from a quick analysis the cost of our platform are much lower than the cost of ELISA for the same antibody target (i.e. Trastuzumab). This is due to the fact that ELISA is not only reagent-intensive but also requires full-time well trained technician. Our platform does not require specialized technicians and experiments are performed using a low-cost, portable potentiostat. To conclude, these advantages make our transduction mechanism suitable for adaptation in point-of-care diagnostics and large-scale high-throughput analysis. More generally, we believe that the design of effective-molarity driven increase of local concentration at the interface with an electrode could represent a valid approach to improve the sensitivity of a number of electrochemical biosensors.

\section{Acknowledgements}

A.P. received support from the University of Rome Tor Vergata under the grant "MIRA" no E81/18000200005. This project has received funding from the European Union's Horizon 2020 research and innovation programme under the Marie Skłodowska-Curie grant agreement Nano-Oligo Med No 778133 M.R. is supported from a Fondazione Umberto Veronesi postdoctoral fellowship. We thank Erica Del Grosso for helpful support with gel electrophoresis experiments.

Keywords: Electrochemical DNA biosensor • antibody monitoring • effective molarity • DNA Nanotechnology •

[1] M. Urdea, L. A. Penny, S. S. Olmsted, M. Y. Giovanni, P. Kaspar, A Shepherd, P. Wilson, C. A. Dahl, S. Buchsbaum, G. Moeller, et al., Nature 2006, 444 Suppl, 73-79.

[2] J. V. Remais, G. Zeng, G. Li, L. Tian, M. M. Engelgau, Int. J. Epidemiol. 2013, 42, 221-227.

[3] P. A. Vieira, C. B. Shin, N. Arroyo-Currás, G. Ortega, W. Li, A. A Keller, K. W. Plaxco, T. E. Kippin, Front. Mol. Biosci. 2019, 6, 69, 1 10.

[4] P. B. Luppa, C. Müller, A. Schlichtiger, H. Schlebusch, TrAC Trends Anal. Chem. 2011, 30, 887-898.

[5] S. Yadav, N. Kashaninejad, M. Kamal, Y. Yamauchi, Biosens. Bioelectron. 2019, 139, 111315

Bioelectron. 2019, 139, 111315 .
D. R. Walt, ACS Nano 2009, 3, 2876-2880.

[6] D. R. Walt, ACS Nano 2009, 3, 2876-2880. Duarte dos Santos, L. H. Marcolino-Junior, M. F. Bergamini, J. Electroanal. Chem. 2019, 842, 140-145.

[8] M. E. Welch, N. L. Ritzert, H. Chen, N. L. Smith, M. E. Tague, Y. Xu, B. A. Baird, H. D. Abruña, C. K. Ober, J. Am. Chem. Soc. 2014, 136, 1879-1883. 
[9] E. Macchia, K. Manoli, B. Holzer, C. Di Franco, M. Ghittorelli, F. Torricelli, D. Alberga, G. F. Mangiatordi, G. Palazzo, G. Scamarcio, et al., Nat. Commun. 2018, 9, 3323, 1-10.

[10] N. Li, L. Liu, M. H. Xiang, J. W. Liu, R. Q. Yu, J. H. Jiang, Chem Commun. 2019, 55, 4387-4390

[11] Z. Chen, C. Wang, L. Hao, R. Gao, F. Li, S. Liu, Biosens. Bioelectron. 2019, 128, 104-112.

[12] K. Tenda, B. van Gerven, R. Arts, Y. Hiruta, M. Merkx, D. Citterio, Angew. Chemie. 2018, 57, 15369-15373.

[13] W. Engelen, L. H. H. Meijer, B. Somers, T. F. A. De Greef, M. Merkx, Nat. Commun. 2017, 8, 14473, 1-8.

[14] R. Arts, I. Den Hartog, S. E. Zijlema, V. Thijssen, S. H. E. Van Der Beelen, M. Merkx, Anal. Chem. 2016, 88, 4525-4532.

[15] L.-P. Qiu, Z.-S. Wu, G.-L. Shen, R.-Q. Yu, Anal. Chem. 2011, 83, 3050-3057.

[16] J. Das, K. B. Cederquist, A. A. Zaragoza, P. E. Lee, E. H. Sargent S. O. Kelley, Nat. Chem. 2012, 4, 642-648.

[17] A. Lass-Napiorkowska, E. Heyduk, L. Tian, T. Heyduk, Anal. Chem. 2012, 84, 3382-3389.

[18] J. Hu, Y. Yu, J. C. Brooks, L. A. Godwin, S. Somasundaram, F. Torabinejad, J. Kim, C. Shannon, C. J. Easley, J. Am. Chem. Soc. 2014, 136, 8467-8474.

[19] A. Bertucci, A. Porchetta, F. Ricci, Anal. Chem. 2018, 90, 1049 1053.

[20] M. Lin, P. Song, G. Zhou, X. Zuo, A. Aldalbahi, X. Lou, J. Shi, C. Fan, Nat. Protoc. 2016, 11, 1244-1263.

[21] T. G. Drummond, M. G. Hill, J. K. Barton, Nat. Biotechnol. 2003, 21, 1192-1199.

[22] Y. Dai, C. C. Liu, Angew. Chem. 2019, 58, 12355-12368.

[23] L. R. Schoukroun-Barnes, F. C. Macazo, B. Gutierrez, J. Lottermoser, J. Liu, R. J. White, Annu. Rev. Anal. Chem. 2016, 9, 163-181.

[24] H. Li, P. Dauphin-Ducharme, G. Ortega, K. W. Plaxco, J. Am Chem. Soc. 2017, 139, 11207-11213.

[25] S. S. Mahshid, F. Ricci, S. O. Kelley, A. Vallée-Bélisle, ACS Sensors 2017, 2, 718-723.

[26] P. Yu, X. He, L. Zhang, L. Mao, Anal. Chem. 2015, 87, 1373-1380.

[27] Y. Lu, X. Li, L. Zhang, P. Yu, L. Su, L. Mao, Anal Chem. 2008, 80 1883-1890.

[28] J. Liu, S. Wagan, M. Dávila Morris, J. Taylor, R. J. White, Anal. Chem. 2014, 86, 11417-11424.

[29] E. E. Ferapontova, Curr. Opin. Electrochem. 2017, 5, 218-225.

[30] E. E. Ferapontova, Annu. Rev. Anal. Chem. 2018, 11, 197-218.

[31] J. Hu, Y. Yu, J. C. Brooks, L. A. Godwin, S. Somasundaram, F. Torabinejad, J. Kim, C. Shannon, C. J. Easley, J. Am. Chem. Soc. 2014, 136, 8467-8474.

[32] S. Liang, A. B. Kinghorn, M. Voliotis, J. K. Prague, J. D. Veldhuis, K. Tsaneva-Atanasova, C. A. McArdle, R. H. W. Li, A. E. G. Cass, W. S. Dhillo, et al., Nat. Commun. 2019, 10, 1-10.

[33] J. Hu, T. Wang, J. Kim, C. Shannon, C. J. Easley, J. Am. Chem. Soc. 2012, 134, 7066-7072.

[34] W. Xu, D. Wang, D. Li, C. C. Liu, Int. J. Mol. Sci. 2019, 21, 134

[35] S. Ranallo, A. Porchetta, F. Ricci, Anal. Chem. 2019, 91, 44-59.

[36] K. W. Plaxco, K. J. Cash, F. Ricci, J. Am. Chem. Soc. 2009, 131, 6955-6957.

[37] D. Kang, C. Parolo, S. Sun, N. E. Ogden, F. W. Dahlquist, K. W. Plaxco, ACS Sensors 2018, 3, 1271-1275.

[38] A. Jemal, E. M. Ward, C. J. Johnson, K. A. Cronin, J. Ma, A. B. Ryerson, A. Mariotto, A. J. Lake, R. Wilson, R. L. Sherman, et al., J. Natl. Cancer Inst. 2017, 109, djx030.

[39] R. J. White, H. M. Kallewaard, W. Hsieh, A. S. Patterson, J. B. Kasehagen, K. J. Cash, T. Uzawa, H. T. Soh, K. W. Plaxco, Anal. Chem. 2012, 84, 1098-1103.

[40] S. S. Mahshid, S. Camiré, F. Ricci, A. Vallée-Bélisle, J. Am. Chem. Soc. 2015, 137, 15596-15599.

[41] S. S. Mahshid, A. Vallée-Bélisle, S. O. Kelley, Anal. Chem. 2017, 89, 9751-9757.

[42] S. S. Mahshid, S. Mahshid, A. Vallée-Bélisle, S. O. Kelley, Anal. Chem. 2019, 91, 4943-4947.

[43] A. A. Rowe, K. N. Chuh, A. A. Lubin, E. A. Miller, B. Cook, D. Hollis,

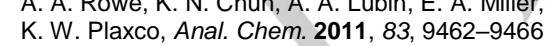

[44] Y. Xiao, A. A. Lubin, B. R. Baker, K. W. Plaxco, A. J. Heeger, Proc. Natl. Acad. Sci. U. S. A. 2006, 103, 16677-16680.

[45] P. Dauphin-Ducharme, K. W. Plaxco, Anal. Chem. 2016, 88 , 11654-11662.

[46] D. Kang, X. Zuo, R. Yang, F. Xia, K. W. Plaxco, R. J. White, Anal. Chem. 2009, 81, 9109-9113.

[47] A. Vallée-Bélisle, F. Ricci, T. Uzawa, F. Xia, K. W. Plaxco, J. Am. Chem. Soc. 2012, 134, 15197-15200.

[48] A. Porchetta, R. Ippodrino, B. Marini, A. Caruso, F. Caccuri, F. Ricci, J. Am. Chem. Soc. 2018, 140, 947-953.

[49] M. Rossetti, R. Ippodrino, B. Marini, G. Palleschi, A. Porchetta, Anal. Chem. 2018, 90, 8196-8201.

[50] E. Golub, H. B. Albada, W. C. Liao, Y. Biniuri, I. Willner, J. Am. Chem. Soc. 2016, 138, 164-172.

[51] B. Z. Stanton, E. J. Chory, G. R. Crabtree, Science 2018, 359 eaao5902

C. S. Sørensen, M. Kjaergaard, Proc. Natl. Acad. Sci. U.S.A. 2019, 116, 23124-23131.

[53] D. Mariottini, A. Idili, M. A. D. Nijenhuis, T. F. A. de Greef, F. Ricci, J. Am. Chem. Soc. 2018, 140, 14725-14734.

[54] L. K. Langeberg, J. D. Scott, Nat. Rev. Mol. Cell Biol. 2015, 16 , 232-244.

[55] E. C. Greenwald, J. M. Redden, K. L. Dodge-Kafka, J. J. Saucerman, J. Biol. Chem. 2014, 289, 2353-2360.

[56] V. M. Krishnamurthy, V. Semetey, P. J. Bracher, N. Shen, G. M. Whitesides, J. Am. Chem. Soc. 2007, 129, 1312-1320.

[57] F. Ricci, R. Y. Lai, A. J. Heeger, K. W. Plaxco, J. J. Sumner, Langmuir 2007, 23, 6827-6834.

[58] R. J. White, N. Phares, A. A. Lubin, Y. Xiao, K. W. Plaxco, Langmuir 2008, 24, 10513-10518.

[59] V. Biagiotti, A. Porchetta, S. Desiderati, K. W. Plaxco, G. Palleschi, F. Ricci, Anal. Bioanal. Chem. 2012, 402, 413-421.

[60] H. W. Schroeder, L. Cavacini, J. Allergy Clin. Immunol. 2010, 125, S41.

[61] Y. Wu, R. D. Tilley, J. J. Gooding, J. Am. Chem. Soc. 2019, 141, $1162-1170$

[62] R. Y. Lai, D. S. Seferos, A. J. Heeger, G. C. Bazan, K. W. Plaxco, Langmuir 2006, 22, 10796-10800.

[63] F. Kuralay, S. Campuzano, J. Wang, Talanta 2012, 99, 155-160.

[64] N. Phares, R. J. White, K. W. Plaxco, Anal. Chem. 2009, 81, 10951100.

G. Chen, I. Dubrawsky, P. Mendez, G. Georgiou, B. L. Iverson, Protein Eng. 1999, 12, 349-356.

[66] S. Y. Tetin, K. M. Swift, E. D. Matayoshi, Anal. Biochem. 2002, 307 84-91.

[67] S. Banala, R. Arts, S. J. A. Aper, M. Merkx, Org. Biomol. Chem. 2013, 11, 7642-7649.

[68] S. Banala, S. J. A. Aper, W. Schalk, M. Merkx, ACS Chem. Biol. 2013, 8, 2127-2132

[69] J. M. Donaldson, C. Zer, K. N. Avery, K. P. Bzymek, D. A. Horne, J. C. Williams, Proc. Natl. Acad. Sci. U. S. A. 2013, 110, 1745617461

[70] B. Jiang, W. Liu, H. Qu, L. Meng, S. Song, T. Ouyang, C. Shou, J. Biol. Chem. 2005, 280, 4656-4662.

[71] V. Demidov, M. D. Frank-Kamenetskii, M. Egholm, O. Buchardt, P. E. Nielsen, Nucleic Acids Res. 1993, 21, 2103-7.

[72] F. Pellestor, P. Paulasova, Eur. J. Hum. Genet. 2004, 12, 694-700.

[73] A. L. Quartino, C. Hillenbach, J. Li, H. Li, R. D. Wada, J. Visich, C. Li, D. Heinzmann, J. Y. Jin, B. L. Lum, Cancer Chemother. Pharmacol. 2016, 77, 77-88. 


\section{Entry for the Table of Contents}
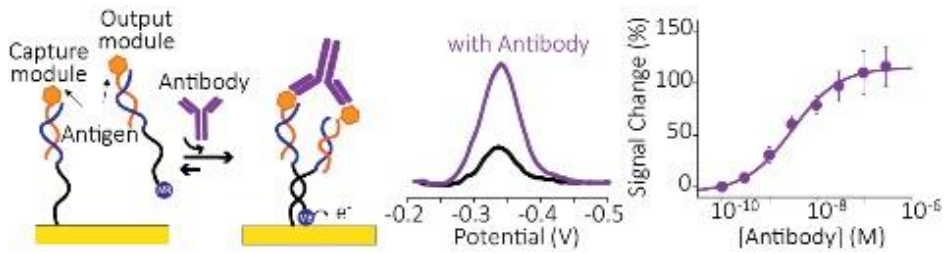

Table of Contents. Antibody detection is crucial for the diagnosis of pathogens and the monitoring of immunotherapy treatments. Here we developed a nucleic acid-based electrochemical platform able to measure immunoglobulins of type $\mathrm{G}$ and $\mathrm{E}$ (IgG and IgE) levels directly in blood serum and other bodily fluids. The antibody detection exploits effective molarity effects that are induced by the spatial confinement of electrochemical active DNA-based sensing modules at the nanoscale. In this work we demonstrate multiplex, rapid and signal-on detection of clinically-relevant antibodies and the potential of the platform for therapeutic drug monitoring applications.

Institute and/or researcher Twitter usernames: @alessan_porch 